\section{Insect integuments}

The Insect Integument. Edited by H. R. Hepburn. Pp. xix +571 . (Elsevier Scientific: Amsterdam, Oxford and New York, 1976.) Dfl.165.00. \$63.50.

This Festschrift is dedicated to A. Glenn Richards, author of the classic Integument of Arthropods (1951) and constructor of the first electron microscope ultramicrotome (1942). In a biographical first chapter, M. Rockstein describes the life and work of 'Glenn', including a bibliography. The miscellany of 26 papers naturally contains much that is already well-known. In the space here available I have therefore selected those items whose originality impressed me.

K. M. Rudall presents excellent Xray diffraction diagrams from cuticles and from Sagitta (Chaetognatha) spines. Had one been more fully Millerindexed, however, it would make the text easier to follow. V. Strout, H. Lipke and T. Geoghegan show by formic acid extraction a narrowing of the range of molecular weights of chitin as the last larval cuticle becomes that of the puparium in flies. This facilitates chitin reorientation at this stage.

In an invigorating chapter, R. A. A. Muzzarelli provides a useful collateral of chemical knowledge from fungal and bacterial research. This includes native chitosan, and the enzymes responsible for its formation from chitin and subsequent degradation. He also reviews chitin-carotenoid linkage in cuticles, and new insecticides acting as inhibitors of chitin synthesis, rightly warning of the danger of their indiscriminate use.

V. B. Wigglesworth emphasises the possible significance of lipids as structural polymers in cuticle. There follows a pioneer attempt (R. H. Hackman) to explain the large number of protein species in cuticles as polymorphism, due to substitution mutations in the second base of genetic code triplets. But the supporting evidence seems to require statistical analysis. S. O. Andersen provides a comprehensive review of crosslinking enzymes both in haemolymph and the cuticle itself. P. Karlson and C. E. Sekeris review the morphogenetic action of ecdysone, augmenting their classical results with recent evidence. P. J. S. Furneaux and A. L. Mackay describe a $90^{\circ}$ cross-ply fibril array in a beetle eggshell, the first in insects, as far as I am aware. R. Dennell revives a mechanistic theory for contol of chitin orientation, but does not mention existing published evidence against it. H. R. Hepburn and I. Joffe have compiled useful and detailed tables of mechanical properties for chitin and whole cuticle. On the basis of stress-strain curves and fractography they distinguish cuticles which show plastic fracture, brittle fracture, or a combination of both. M. Locke relates cuticle formation to alternate secretion by plasma membrane plaques and Golgi vesicles.

S. Caveney presents results which may shed light on the unsolved problem of cell morphogenetic gradients. He shows that insect epidermis functions as a syncytium by cell/cell communication via gap junctions. The late J. M. Whitten records the regular orientation of centriole-like structures in the cells of fly feet, and suggests that they may be related to formation of regular hair patterns. E. P. Marks and B. A. Sowa are successfully exploiting in vitro cuticle growth methods for investigating blocks to chitin synthesis and effects of insecticides on cuticle formation under carefully controlled conditions. Chapters by $\boldsymbol{H}$. Nemenz and W. Ebeling both stress the importance of epidermis rather than epicuticle as a water-controlling mechanism in relation to osmoregulation and insecticides respectively.

J. F. V. Vincent compares stresssoftening in locust intersegmental membrane and filled rubber composites,

\section{Atmospheric radiative transfer}

Radiative Processes in Meteorology and Climatology. (Developments in Atmospheric Science, Vol. 5.) By G. W. Paltridge. Pp. xvii +318 . (Elsevier Scientific: Amsterdam, Oxford and New York, 1976.) Dfl.103; $\$ 39.75$.

THE basic atmospheric engine operates between a source which is derived from solar radiation absorbed by the atmosphere, or by the surface, and a sink which is the emission of infrared radiation to space. Radiation processes are, therefore, of fundamental importance to atmospheric study. Further, because of the current concern about the global climate and possible mechanisms through which climate may change, there is a lot of interest in the Earth's radiation budget and the possibility of small alterations which might occur in it, due to changes in the radiation input or output. For instance, variations in the net solar input might occur either due to changes in the sun itself or changes in the atmospheric cloud or dust content; or variations in the emitted radiation from the atmosphere itself might occur for similar reasons.

There are few textbooks available on atmospheric radiative transfer. One of the best of the standard texts written by R. M. Goody in 1964 is now out of print, so that this new text while H. C. Bennet-Clark provides a lucid account of jumping principles and cuticular energy storage mechanisms. F. G. Barth summarises results of photoelastic analysis of strains applied to perspex models of the cuticular parts of arachnid mechanoreceptors. Ablation experiments have pinpointed the specific sense organs predicted to be responsible for the idiothetic sense of spiders. This is the sense of orientation using the experience of previous movement sequences, without using landmarks. H. E. Hinton reviews a wide range of recent work on insect physical colour systems. He records anti-reflection nipples (previously thought to be restricted to corneal surfaces) on the wing scales of the green hairstreak butterfly. W. Kenchington presents an intriguing range of modes of formation of peritrophic membranes. W. Peters summarises recent results on in vitro growth of peritrophic membrane, and factors which affect it.

The volume is well produced and will clearly be necessary reading for relevant workers-if their institutions can afford it.

A. C. Neville

Dr Neville is a Reader in Zoology at the University of Bristol, UK.

by Paltridge and Platt is very much to be welcomed. It presents the fundamental theory of radiative transfer, and shows how empirical and theoretical approximations may be applied to the transfer of radiation in the clear atmosphere and in clouds. A lot of very useful up-to-date information is contained in the book about the radiative properties of the surface, of different types of clouds and aerosol, and about atmospheric transmission in different spectral regions. Emphasis is placed on radiative processes in the troposphere; little is said about the high atmosphere.

The book does not set out to be a thorough theoretical treatise. In fact, some of the theoretical presentation is confused (for instance, the presentation of the Curtis-Godson approximation in chapter 7 and its confusion with the pressure scaling of absorber amount). Rather, the book is aimed at providing the methods and means for meteorologists and atmospheric physicists who need to incorporate radiation processes into atmospheric models or work out simple practical atmospheric problems involving radiative transfer. For such people the book will be very valuable indeed and well worth purchasing. J. T. Houghton

I. T. Houghton is Professor in the Department of Atmospheric Physics at the Clarendon Laboratory, University of Oxford, UK. 